\title{
Investigation of Filamentous Basidiomycetes in the Airway Is the Third Unmet Need in the Management of Unexplained Chronic Cough in Adults
}

\author{
Haruhiko Ogawa $^{a}$ Kazuya Tone ${ }^{b}$ Koichi Makimurac \\ aDepartment of Environmental and Preventive Medicine, Kanazawa University, \\ Kanazawa, Japan; ${ }^{b}$ Division of Respiratory Medicine, Jikei University School of Medicine, \\ Tokyo, Japan; 'Laboratory of Space and Environmental Medicine, Teikyo University, \\ Tokyo, Japan
}

\begin{abstract}
What Is It about?
Filamentous basidiomycetes (f-BM) that has been recognized as an exacerbation factor of cough symptoms were cultured from the sputum of 91 patients (79.8\%) with unexplained chronic cough (UCC). The fractional exhaled nitric oxide level was significantly different between patients with and without f-BM colonization ( 23.0 vs. 15.2, respectively). UCC patients with f-BM colonization were diagnosed as having fungus-associated chronic cough and showed a good clinical response to antifungal drugs. Lack of examination of f-BM in the sputum is one factor leading to the overlooking of treatable underlying causes that are falsely labeled as unexplained.
\end{abstract}

Dear Editor,

Managing unexplained chronic cough (UCC) is still an important issue even among cough specialists. Irwin et al. [1] commented on two clinical needs that must be met to improve quality of life in patients with UCC: the development of new therapies and the need for clinicians to practice intervention fidelity by adhering to best clinical practice guidelines for chronic cough. Here, we discuss a third unmet need related to environmental assessment, which should be included in "further investigation to consider" described in the clinical practice guidelines for chronic cough [2].

Although filamentous basidiomycetes (f-BM), environmental fungi, are not generally detected in airway specimens of respiratory health patients, f-BM colonization in the airway mucosa of chronic cough patients has been recognized as an exacerbation factor of cough symptoms [3]. Airway mucus plugs, recently reported to exacerbate asthma, have also been detected in the peripheral airways of patients with chronic cough with f-BM colonization [4]. The relation between f-BM colonization and mucus plug formation in the airway is an important concern. 
Biomedicine Hub

\begin{tabular}{l|l}
\hline Biomed Hub 2020;5:508611 \\
\hline DOI: 10.1159/000508611 & $\begin{array}{l}\text { (c) 2020 The Author(s). Published by S. Karger AG, Basel } \\
\text { www.karger.com/bmh }\end{array}$ \\
\hline Ogawa et al.: Filamentous Basidiomycetes in the Management of Unexplained Chronic
\end{tabular}

Ogawa et al.: Filamentous Basidiomycetes in the Management of Unexplained Chronic Cough is

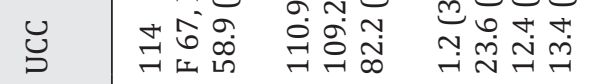

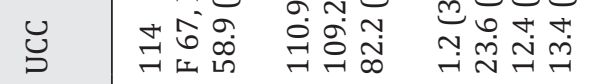

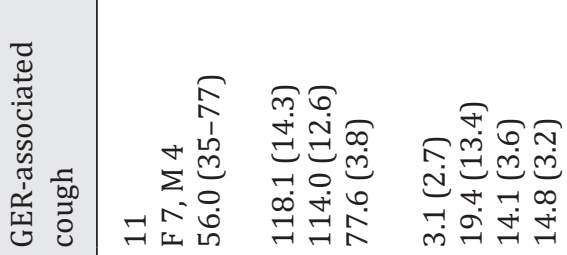
(1)

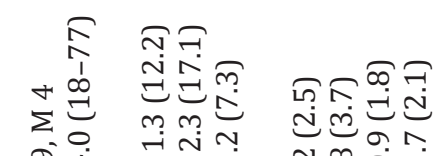

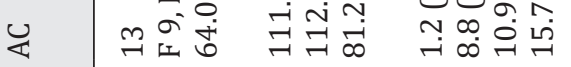

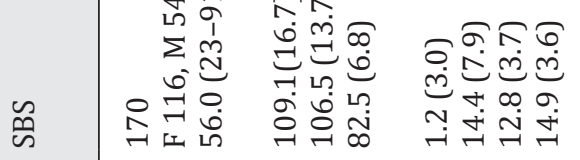

ผे สิ กิ

๙

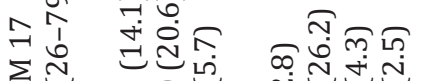

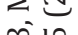

m

L $\underbrace{}_{0}$ vi

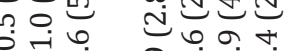

a.

(1)

$\dot{m} \stackrel{+}{\neg}$

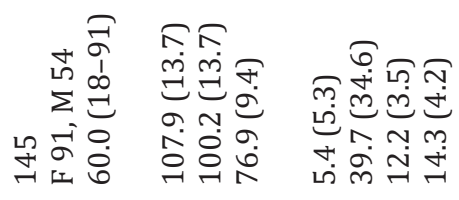

约

(1)
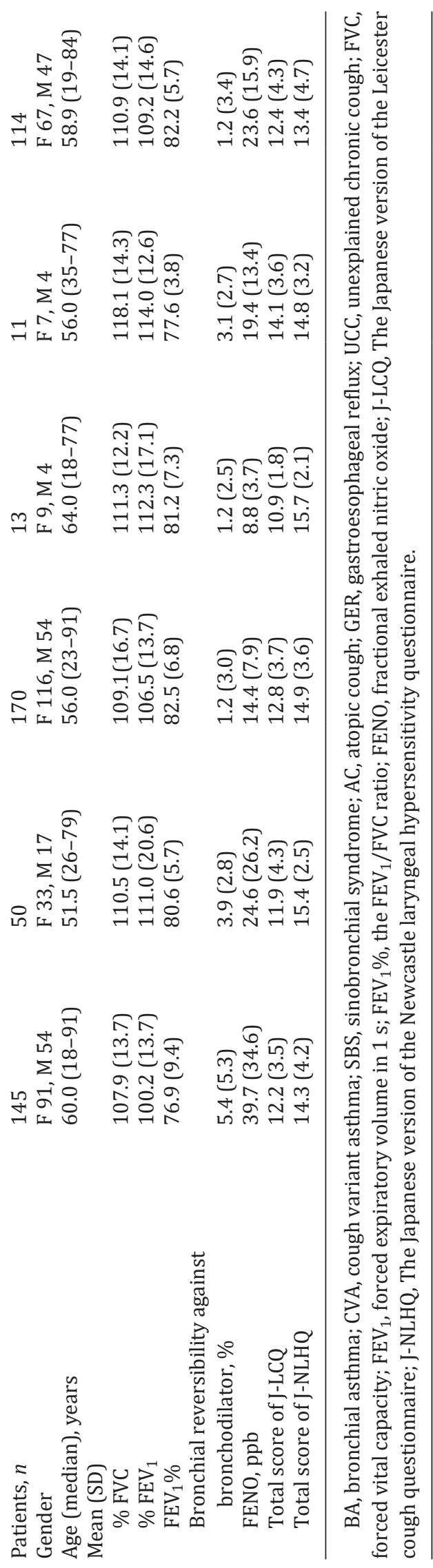
Ogawa et al.: Filamentous Basidiomycetes in the Management of Unexplained Chronic Cough

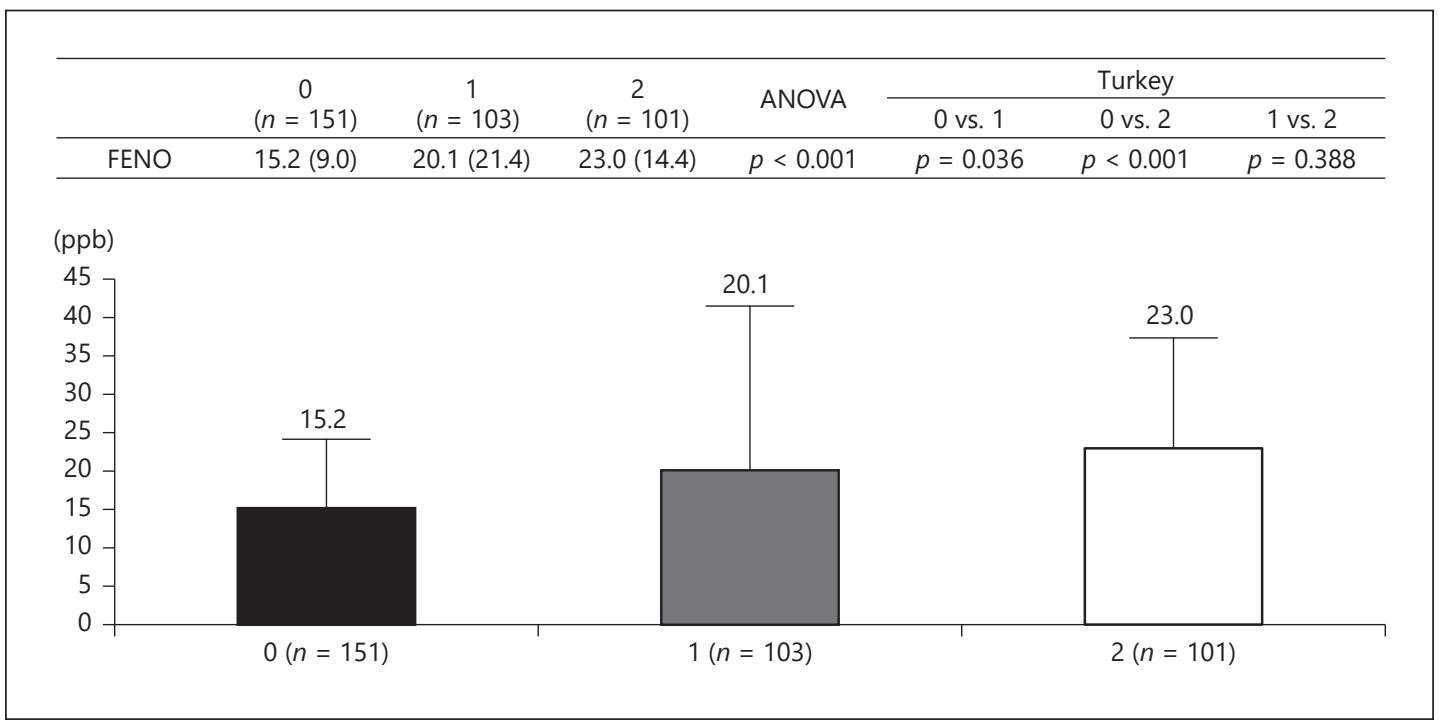

Fig. 1. The level of fractional exhaled nitric oxide (FENO) was significantly higher in the f-BM culture-positive group than the culture-negative group. Group 0: culture-negative group; Group 1: other fungal culture-positive group; Group 2: f-BM culture-positive group.

Of 555 patients visiting related facilities and our hospital for diagnosis and treatment of chronic intractable cough between January 2017 and December 2019, retrospective analysis was performed in 503 patients (51 patients were excluded) (online suppl. material; for all online suppl. material, see www.karger.com/doi/10.1159/000508611). After excluding bronchial asthma (BA), cough variant asthma, sinobronchial syndrome, and gastroesophageal reflux-associated cough in accordance with clinical practice guidelines of chronic cough, 114 patients were initially diagnosed with UCC (Table 1).

f-BM were isolated and cultured from the sputum of $138(28.2 \%)$ of 490 patients undergoing sputum fungal culture examination and were detected in the sputum of 91 (79.8\%) UCC patients. Excluding patients with BA, which often shows an elevated fractional exhaled nitric oxide (FENO) level, the FENO level was significantly different between patients with and without BM colonization (23.0 [SD 14.4] vs. 15.2 [9.0], respectively) (Fig. 1). The mechanisms by which BM colonization influences the FENO level is unclear. However, these results suggest the necessity of reconsidering the interpretation of FENO in allergic airway disorders [5].

UCC patients with BM colonization showing a good clinical response to antifungal drugs were diagnosed as having fungus-associated chronic cough (FACC), which has recently been introduced in the Japanese Respiratory Society Guidelines for the Management of Cough and Sputum 2019 [6, 7], and such patients were excluded from initial diagnosis of UCC [8]. In our preliminary studies, central suppressant therapies, such as pregabalin or gabapentin, were not always more efficacious on cough symptoms of FACC patients than antifungal drugs [9]. Therefore, it is of concern whether new therapies, such as the P2X3 receptor antagonist, gefapixant, will show sufficient efficacy on cough symptoms in refractory cough patients with f-BM colonization.

Lack of examination of f-BM in the sputum of UCC patients is one factor leading to the overlooking of treatable underlying causes that are falsely labeled as unexplained. As culturing and identification of f-BM in bronchial specimens from UCC patients are technically difficult using current methods, fungal culture examination has not been widely used. The development of culture plates for selection of f-BM will facilitate multicenter clinical research regarding this third unmet need in addressing UCC [1]. 


\section{Conflicts of Interest Statement}

The authors report no potential financial disclosure or conflicts of interest.

\section{Author Contributions}

K.M. takes responsibility for the content of the manuscript including the data and analysis. H.O. and K.T. contributed substantially to the study design, data analysis and interpretation, and the writing of the manuscript.

\section{References}

1 Irwin RS, French CL, Madison JM. Managing unexplained chronic cough in adults: what are the unmet needs? Lancet Respir Med. 2020 Feb 25;S2213-2600(20)30083-7.

2 Irwin RS, French CL, Chang AB, Altman KW, Adams TM, Altman KW, et al.; CHEST Expert Cough Panel*. Classification of Cough as a Symptom in Adults and Management Algorithms: CHEST guideline and expert panel report. Chest. 2018 Jan;153(1):196-209.

3 Chowdhary A, Agarwal K, Meis JF. Filamentous Fungi in Respiratory Infections. What Lies Beyond Aspergillosis and Mucormycosis? PLoS Pathog. 2016 Apr;12(4):e1005491.

4 Okumura K, Ogawa H, Yoshie Y, Nadamura T, Igarashi T, Tone K, et al. Mucus plugs and bronchial wall thickening on three-dimensional computed tomography in patients with unexplained chronic cough whose sputum yielded filamentous Basidiomycetes. Eur Radiol. 2020 Jun;30(6):3268-76.

5 Ogawa H, Fujimura M, Ohkura N, Satoh K, Makimura K. Fungus-associated asthma: overcoming challenges in diagnosis and treatment. Expert Rev Clin Immunol. 2014 May;10(5):647-56.

6 Ogawa H, Fujimura M, Takeuchi Y, Makimura K. Efficacy of itraconazole in the treatment of patients with chronic cough whose sputa yield basidiomycetous fungi-fungus-associated chronic cough (FACC). J Asthma. 2009 May; 46(4):407-12.

7 Tone K, Ogawa H, Alshahni MM, Kuwano K, Makimura K. Real-Time PCR Detection of the Basidiomycetous Fungus Bjerkandera adusta: A Tool to Identify Itraconazole Responder Patients with Unexplained Chronic Cough. Respiration. 2019;97(1):84-91.

8 Ogawa H, Fujimura M, Ohkura N, Makimura K. It is time to call attention to the clinical significance of fungal colonization in chronic cough. Allergol Int. 2014 Dec;63(4):611-2.

9 Ogawa H, Tone K, Fujimura M, Makimura K. Central suppressant therapies in unexplained chronic cough patients whose sputum cultures yielded Bjerkandera adusta. Allergol Int. 2019 Jan;68(1):125-6. 